\title{
Effect of ART1 on the proliferation and migration of mouse colon carcinoma CT26 cells in vivo
}

\author{
JIAN-XIA XU* ${ }^{*}$ WEI XIONG ${ }^{*}$, ZHEN ZENG ${ }^{*}$, YI TANG, YA-LAN WANG, \\ MING XIAO, MING LI, QING SHU LI, GUANG-LIN SONG and JING KUANG \\ Department of Pathology, Molecular Medicine and Cancer Research Center, \\ Chongqing Medical University, Chongqing 400016, P.R. China
}

Received November 29, 2015; Accepted November 17, 2016

DOI: $10.3892 / \mathrm{mmr} .2017 .6152$

\begin{abstract}
Arginine-specific mono-ADP-ribosyltransferase 1 (ART1) is an important enzyme that catalyzes arginine-specific mono-ADP-ribosylation. There is evidence that arginine-specific mono-ADP-ribosylation may affect the proliferation of smooth muscle cells via the Rho-dependent signaling pathway. Previous studies have demonstrated that ART1 may have a role in the proliferation, invasion and apoptosis of colon carcinoma in vitro. However, the effect of ART1 on the proliferation and invasion of colon carcinoma in vivo has yet to be elucidated. In the present study, mouse colon carcinoma CT26 cells were infected with a lentivirus to produce ART1 gene silencing or overexpression, and were then subcutaneously transplanted. To observe the effect of ART1 on tumor growth or liver metastasis in vivo, a spleen transplant tumor model of CT26 cells in BALB/c mice was successfully constructed. Expression levels of focal adhesion kinase (FAK), Ras homolog gene family member A (RhoA) and the downstream factors, c-myc, c-fos and cyclooxygenase-2 (COX-2) proteins, were measured in vivo. The results demonstrated that ART1 gene silencing inhibited the growth of the spleen transplanted tumor and its ability to spread to the liver via metastasis. There was also an accompanying increase in expression of FAK, RhoA, c-myc, c-fos and COX-2, whereas CT26 cells with ART1 overexpression demonstrated the opposite effect. These results suggest a potential role for ART1 in
\end{abstract}

Correspondence to: Professor Ya-Lan Wang, Department of Pathology, Molecular Medicine and Cancer Research Center, Chongqing Medical University, 1 Medical College Road, Yuzhong, Chongqing 400016, P.R. China

E-mail: wangyalan074@126.com

*Contributed equally

Key words: arginine-specific mono-ADP-ribosyltransferase 1, colon carcinoma, proliferation, migration, RhoA, focal adhesion kinase, in vivo study the proliferation and invasion of CT26 cells and a possible mechanism in vivo.

\section{Introduction}

Adenosine diphosphate (ADP) ribosylation, which includes mono-ADP-ribosylation, poly-ADP-ribosylation, ADP-ribose cyclization and formation of O-acetyl-ADP-ribose, is involved in a wide range of human physiological and pathological processes and serves important roles in cell signal transduction, transcriptional regulation, genetic stability maintenance, cell proliferation and differentiation, adhesion and migration (1). Mono-ADP-ribosyltransferases (ART), the enzymes of mono-ADP-ribosylation, consist of seven members (ART1-7). ART1 catalyzes the mono-ADP-ribosylation of nicotinamide adenine dinucleotide to arginine residues in proteins, thereby releasing nicotinamide, which may alter the structure and chemical property of acceptor proteins resulting in a change in their activity and function (2). Research on ART1 is mainly concentrated on the inflammatory response and on non-neoplastic cells $(3,4)$. In the epithelial cells of the respiratory tract and the bronchoalveolar lavage fluid of people with asthma, ART1 may catalyze the mono-ADP-ribosylation of human neutrophil peptide-1 (3), resulting in an inflammatory response. Yau et al (5) demonstrated that meta-iodobenzylguanidine (MIBG), a selective inhibitor of arginine-specific mono-ADP-ribosylation (6), is able to suppress the proliferation and differentiation of vascular smooth muscle cells. The researchers hypothesized that mono-ADP-ribosylation is involved in a Rho-dependent signaling pathway.

However, although ART1 is associated with the proliferation and migration of colon cancer cells in vivo, its molecular mechanism has yet to be fully elucidated. In the present study, mouse colon carcinoma CT26 cells were infected with a lentivirus to change the expression of ART1 in CT26 cells. To observe the effect of ART1 on the development of colon carcinoma in vivo, CT26 cells with ART1 silencing or overexpression were injected into $\mathrm{BALB} / \mathrm{c}$ mice to construct a subcutaneously transplanted tumor model or a spleen transplant tumor model. Growth of the tumor and liver metastases were observed. In addition, the expression of focal adhesion kinase (FAK), Ras homolog gene family member A (RhoA) and their downstream factors, c-myc, c-fos and cyclooxygenase-2 
(COX-2) proteins, were measured. The potential role of ART1 in the proliferation and invasion of CT26 cells and its possible mechanism in vivo were explored.

\section{Materials and methods}

Cell lines and animals. The mouse colon adenocarcinoma CT26 cell line was obtained from Professor Yu-Quan Wei (Sichuan University, Chengdu, Sichuan, China), Tang et al and Kuang et al $(7,8)$, having successfully constructed ART1-short hairpin RNA (shRNA), ART1-overexpression and vector-control CT26 cells. BALB/c mice (6-8 weeks old, 18-22 g) were obtained from the animal experimental center of Chongqing Medical University (Chongqing, China) and placed in the specific pathogen-free feeding room $\left(20-26^{\circ} \mathrm{C}\right.$, $12 \mathrm{~h}: 12 \mathrm{~h}$ light/dark cycle) of the animal experimental center at Chongqing Medical University.

Subcutaneously transplanted tumor model of CT26 cells in $B A L B / c$ mice. Each experimental group consisted of 12 mice. Each mouse was anesthetized by the intraperitoneal injection of $2 \%$ chloral hydrate $(0.3 \mathrm{~g} / \mathrm{kg})$. CT26 cell suspension $\left(1 \times 10^{7} / \mathrm{mlx} 50 \mu \mathrm{l}\right)$ was subcutaneously injected into the lateral skin of the right armpit of each mouse. After 14 days, six mice were randomly selected from each group for sacrifice, and the weight and volume of the subcutaneous tumor was recorded. The survival time of the rest of the mice in each group was recorded. Tumor volume was calculated according to the formula: Volume=the maximum diameter $\mathrm{x}$ the most trails ${ }^{2} \times 1 / 2(9)$.

Spleen transplant tumor model of CT26 cells in BALB/c mice to observe liver metastases. A total of $48 \mathrm{BALB} / \mathrm{c}$ mice were randomly divided into four groups. Following the method described by Liu et al (10), each mouse was anesthetized with $2 \%$ chloral hydrate $(0.015 \mathrm{ml} / \mathrm{g})$ injected into the abdominal cavity. Subsequently, the abdominal wall was incised along with the left subcostal margin layer by layer. The spleen was identified in the abdominal cavity, and then CT26 cell suspension $\left(1 \times 10^{7} / \mathrm{ml} \times 50 \mu \mathrm{l}\right)$ was injected under the capsule of the spleen. Finally, the abdominal wall was sutured. The entire procedure was performed under sterile conditions to ensure the survival rate of the mice. While being reared the mice were provided with standard chow and tap water ad libitum. After 14 days, six mice were randomly selected from each group for sacrifice and the remaining mice of each group continued to be fed until their natural death to enable the recording of the survival time and the plotting of a Kaplan-Meier survival curve.

The volume of the spleen tumors was calculated according to the formula volume $=$ the maximum diameter $\mathrm{x}$ the most trails $^{2} \times 1 / 2(9)$. Nodules of liver metastases were graded as follows: Grade 0, no visible metastatic nodule in liver; grade 1, 1-5 metastatic nodules in liver; grade 2, 6-10 metastatic nodules in liver; grade 3,>10 metastatic nodules, or fused nodules difficult to count exactly (10).

Expression levels of ART1, RhoA, c-myc, c-fos and COX-2 detected with western blotting in the subcutaneously transplanted tumor. The subcutaneous tumors were cut into small pieces, weighed, homogenized, and then lysed with radio-immunoprecipitation assay (RIPA) lysis buffer (100 $\mu \mathrm{l}$ of RIPA lysis buffer/10 mg tissue; Beyotime Institute of Biotechnology, Shanghai, China) for $30 \mathrm{~min}$ on ice. The lysate was transferred into a $1.5 \mathrm{ml}$ centrifuge tube and centrifuged at $4^{\circ} \mathrm{C} 12,000 \mathrm{rpm}(8,418 \mathrm{~g})$ for $5 \mathrm{~min}$. A bicinchoninic acid (BCA) protein assay kit (Beyotime Institute of Biotechnology) was used to measure the concentration of protein. Protein (80 $\mu \mathrm{g}$ /lane) was electrophoresed on $10 \%$ polyacrylamide gels (SDS-PAGE) and then transferred to polyvinylidene fluoride (PVDF) membranes. The membranes were blocked with 5\% non-fat dried milk dissolved in Tris-buffered saline with Tween-20 (TBST) at room temperature for $2 \mathrm{~h}$, and incubated respectively with primary antibodies of ART1 (cat. no. AP2311a; Abgent, Inc., San Diego, CA, USA), RhoA (cat. no. BS6470), and c-fos (cat. no. BS6433; Bioworld Technology, Inc., St. Louis, MO, USA), c-myc (cat. no. C10262; Anbo, Inc., San Francisco, CA, USA), COX-2 (cat. no. 12375-1-AP; Proteintech Group, Inc., Chicago, IL, USA) and $\beta$-actin (cat. no. BA2305; Boster Systems, Wuhan, China) overnight at $4^{\circ} \mathrm{C}$. The most effective working concentration of these primary antibodies was 1:500. The membranes were washed three times with TBST, and then incubated with horseradish peroxidase-conjugated goat anti-rabbit $\operatorname{IgG}$ secondary antibody at a dilution of 1:1,000 (ZSGB-BIO, Beijing, China) for $1.5 \mathrm{~h}$ at room temperature. The membranes were washed three times with TBST, and then dipped into BeyoECL Plus (Beyotime Institute of Biotechnology, Shanghai, China) for exposure and imaging (Bio-Rad Laboratories, Inc., Hercules, CA, USA). $\beta$-actin was used as a loading control for the western blotting experiments.

Western blot analysis of expression levels of ART1, RhoA and FAK in transplanted spleen tumors. Total protein was extracted from transplanted spleen tumors. The tissue was washed with phosphate-buffered saline (PBS) and then homogenized prior to being lysed with RIPA lysis buffer $(100 \mu 1 / 10 \mathrm{mg})$ for $30 \mathrm{~min}$ on ice. The homogenate was transferred to a pre-cooled centrifuge tube, and then centrifuged at $4^{\circ} \mathrm{C}, 12,000 \mathrm{rpm}(8,418 \mathrm{~g})$ for $10 \mathrm{~min}$. The rest of the procedure was as detailed in the previous paragraph with the exception that the primary antibodies, ART1 (Abgent, Inc.), RhoA and FAK (cat. no. BS6899; Bioworld Technology, Inc.) at a dilution of 1:500, and $\beta$-actin (Boster Systems) at a dilution of 1:1,000 were used to incubate the PVDF membranes.

Statistical analysis. Data were presented as the mean \pm standard deviation. Analysis of variance statistical evaluation was used and analyses were performed using SPSS software, version 17.0 (SPSS, Inc., Chicago, IL, USA). The Kruskal-Wallis and Nemenyi methods were used to analyze the level of metastatic nodules in the liver. The differences in tumor-bearing mice survival time were analyzed using the log-rank test. $\mathrm{P}<0.05$ was considered to indicate a statistically significant difference.

\section{Results}

Effect of ART1 on the growth of subcutaneous transplanted CT26 tumors in BALB/c mice. Compared with subcutaneous 


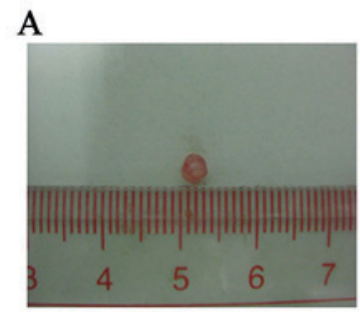

ART1-shRNA

B

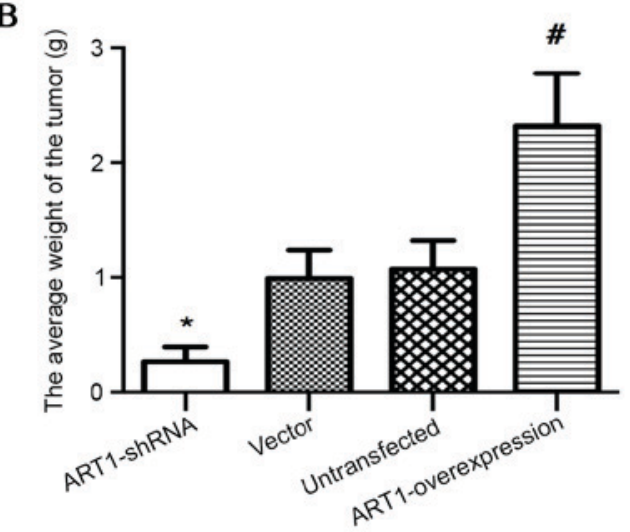

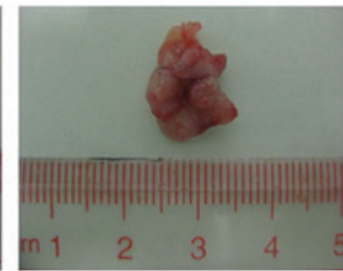

Vector

C

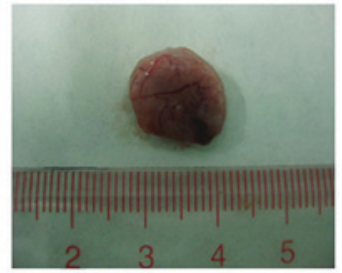

Untransfected

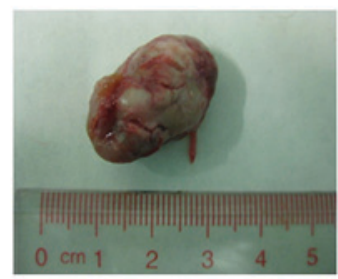

ART1-overexpression

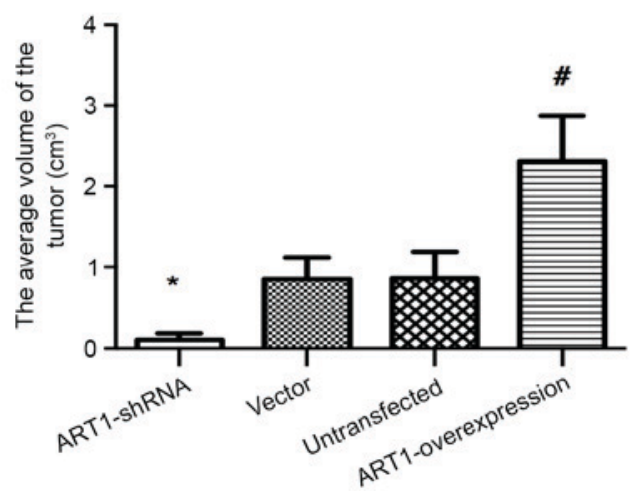

Figure 1. Subcutaneously transplanted CT26 tumors. (A) Photographic images of subcutaneously transplanted CT26 tumors in BALB/c mice are shown. The (B) average weight and (C) volume of subcutaneous transplanted tumors in the four groups. "P<0.05, ART1-shRNA group vs. vector and untransfected groups; ${ }^{\#} \mathrm{P}<0.05$, ART1-overexpression group vs. vector and untransfected groups. ART1-shRNA, ADP-ribosyltransferase 1-short hairpin RNA.

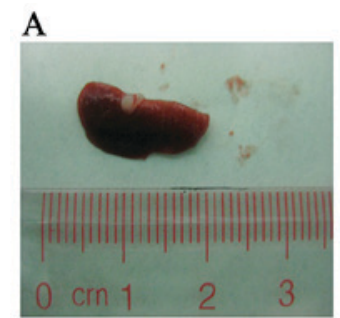

ART1-shRNA

B

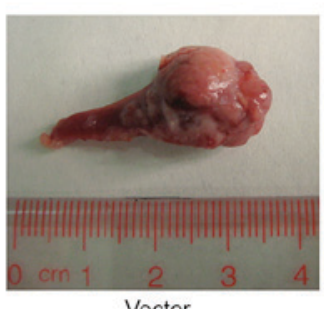

Vector

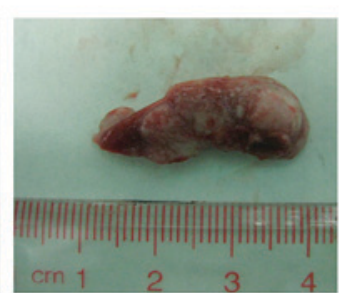

Untransfected

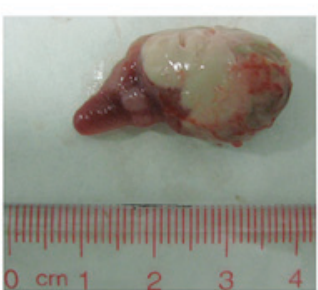

ART1-overexpression

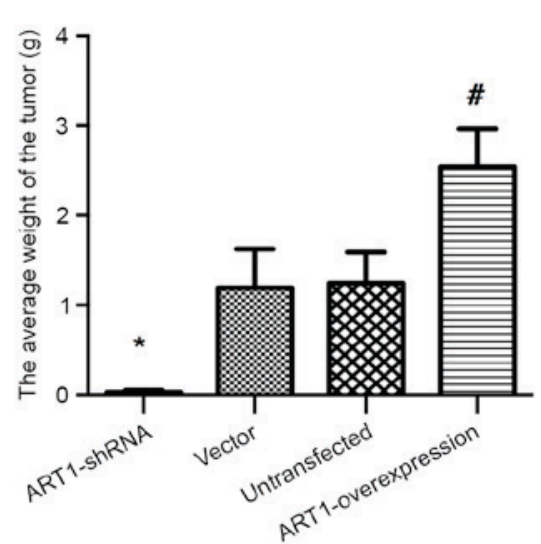

C

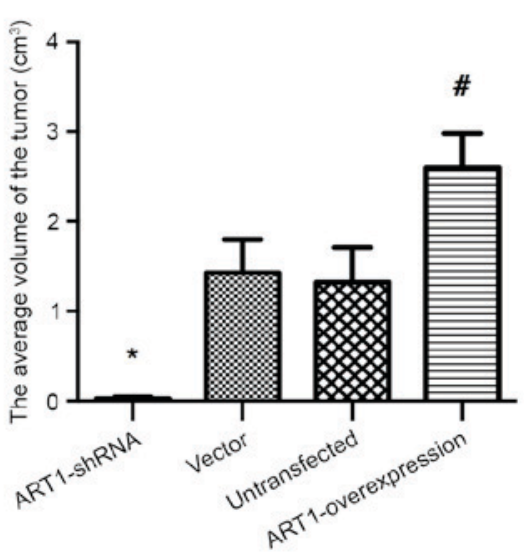

Figure 2. Spleen-transplanted CT26 tumors. (A) Spleen-transplanted CT26 tumors in BALB/c mice are shown. Comparison of the (B) weight and (C) volume of spleen-transplanted tumors in the four groups. ${ }^{*} \mathrm{P}<0.05$, ART1-shRNA group vs. vector and untransfected groups; ${ }^{\#} \mathrm{P}<0.05$, ART1-overexpression group vs. vector and untransfected groups. ART1-shRNA, ADP-ribosyltransferase 1-short hairpin RNA.

transplanted vector-control and untransfected CT26 tumors, the volume and weight of subcutaneous transplanted tumors were decreased in the ART1-shRNA group $(\mathrm{P}<0.05)$ and increased in the ART1-overexpression group $(\mathrm{P}<0.05$; Fig. 1A-C).
Effects of ART1 on the growth of spleen transplanted CT26 tumor in BALB/c mice. The volume and weight of spleen-transplanted ART1-shRNA CT26 tumors were significantly decreased compared with the spleen-transplanted vector-control and untransfected CT26 tumors $(\mathrm{P}<0.05)$. 
Table I. The quantity and grading of metastases in liver ( $n=6$, mean \pm standard deviation).

\begin{tabular}{lccrr}
\hline & & & Grade \\
\cline { 3 - 5 } Group & Quantity of metastases in the liver & 0 & 1 & 2 \\
\hline ART1-shRNA & $0.33 \pm 0.82^{\mathrm{a}}$ & 5 & 1 & 0 \\
Vector & $22.33 \pm 12.11$ & 0 & 0 & 0 \\
Untransfected & $23.83 \pm 10.340$ & 0 & 0 & 1 \\
ART1-overexpression & $39.50 \pm 8.38^{\mathrm{b}}$ & 0 & 0 & 0 \\
\hline
\end{tabular}

${ }^{\mathrm{a}} \mathrm{P}<0.05$, ART1-shRNA group vs. vector and untransfected groups; ${ }^{\mathrm{b}} \mathrm{P}<0.05$, ART1-overexpression group vs. vector and untransfected groups ART1-shRNA, ADP-ribosyltransferase 1-short hairpin RNA.

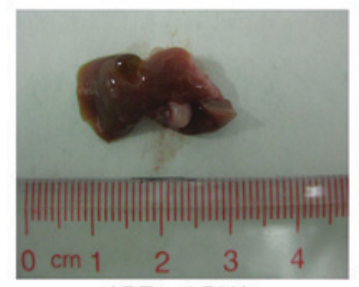

ART1-shRNA

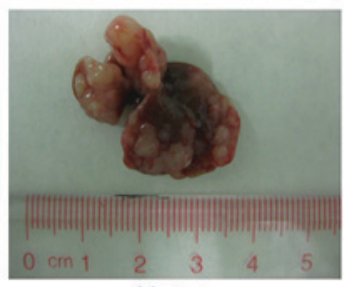

Vector

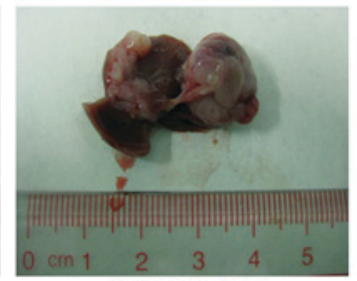

Untransfected

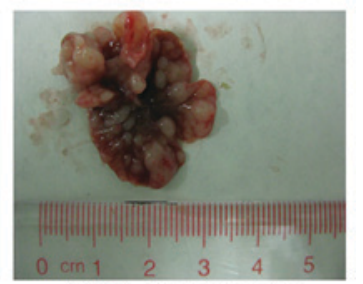

ART1-overexpression

Figure 3. Liver metastasis of colon carcinoma in BALB/c mice. ART1-shRNA, ADP-ribosyltransferase 1-short hairpin RNA.

However, the volume and weight of spleen-transplanted ART1-overexpression CT26 tumors were increased $(\mathrm{P}<0.05)$. No significant differences were identified between the spleen-transplanted vector-control and untransfected CT26 tumors ( $\mathrm{P}>0.05$; Fig. 2A-C).

Effect of ART1 on liver metastasis of colon carcinoma in $B A L B / c$ mice. The number of liver metastatic tumor nodules in the spleen-transplanted CT26 tumor model were counted in each group. The number of liver metastatic tumor nodules in the ART1-shRNA group was lower than in the vector-control and untransfected groups $(\mathrm{P}<0.05)$. The number of liver metastatic tumor nodules in the ART1-overexpression group was higher compared with the vector-control and untransfected groups $(\mathrm{P}<0.05)$. No significant differences were identified in the number or the appearance of liver metastatic tumor nodules in the vector-control and untransfected groups $(\mathrm{P}>0.05$; Fig. 3 and Table I).

Influence of ART1 on the survival time of $B A L B / c$ mice with subcutaneous transplanted CT26 tumor or spleen transplanted CT26 tumor. The average survival time of BALB/c mice with subcutaneously transplanted CT26 tumors was extended in the ART1-shRNA group $(\mathrm{P}<0.05)$, and was shortened in the ART1-overexpression group $(\mathrm{P}<0.05)$. However, no significant differences were identified between the vector-control and untransfected groups ( $\mathrm{P}>0.05$; Fig. 4A).

The average survival time of $\mathrm{BALB} / \mathrm{c}$ mice with spleen-transplanted ART1-shRNA CT26 tumors was longer compared with vector-control and untransfected groups $(\mathrm{P}<0.05)$. The average survival time of BALB/c mice with spleen-transplanted ART1-overexpression CT26 tumors was shorter than in the control groups $(\mathrm{P}<0.05)$. No significant
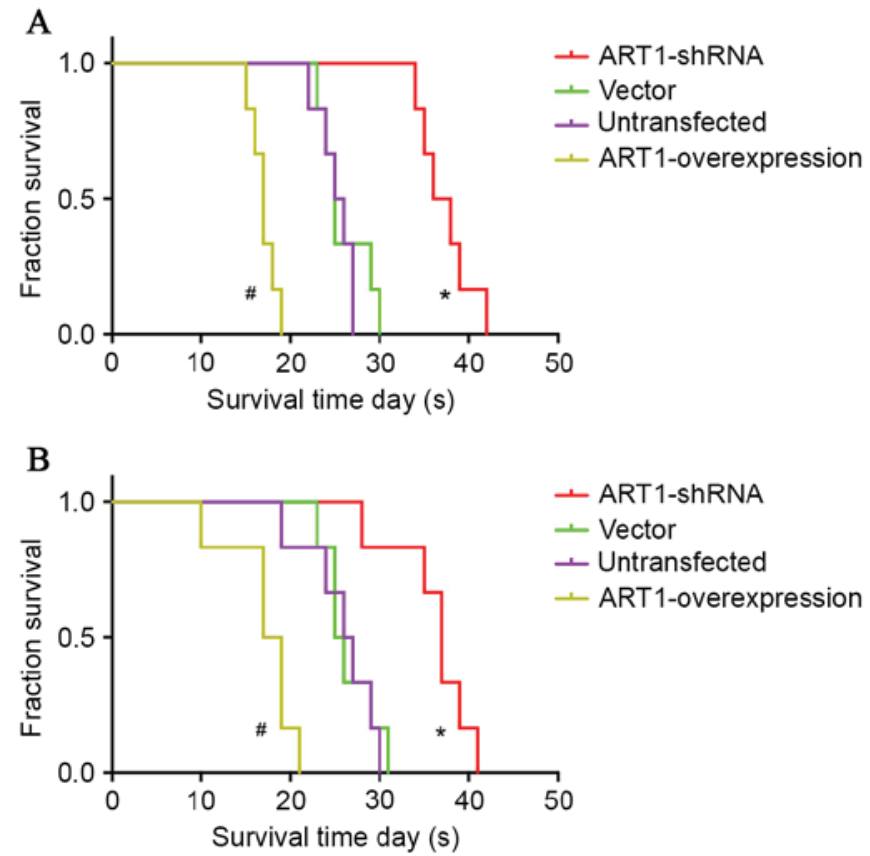

Figure 4. Kaplan-Meier survival curves of BALB/c mice with (A) subcutaneously transplanted or (B) spleen-transplanted CT26 tumors. ${ }^{*} \mathrm{P}<0.05$, ART1-shRNA group vs. vector and untransfected groups; ${ }^{\#} \mathrm{P}<0.05$, ART1-overexpression group vs. vector and untransfected groups. ART1-shRNA, ADP-ribosyltransferase 1-short hairpin RNA.

differences were identified between the vector-control and untransfected groups ( $\mathrm{P}>0.05$; Fig. 4B).

Effect of ART1 on the expression levels of RhoA, c-myc, c-fos and COX-2 in subcutaneously transplanted CT26 tumor 

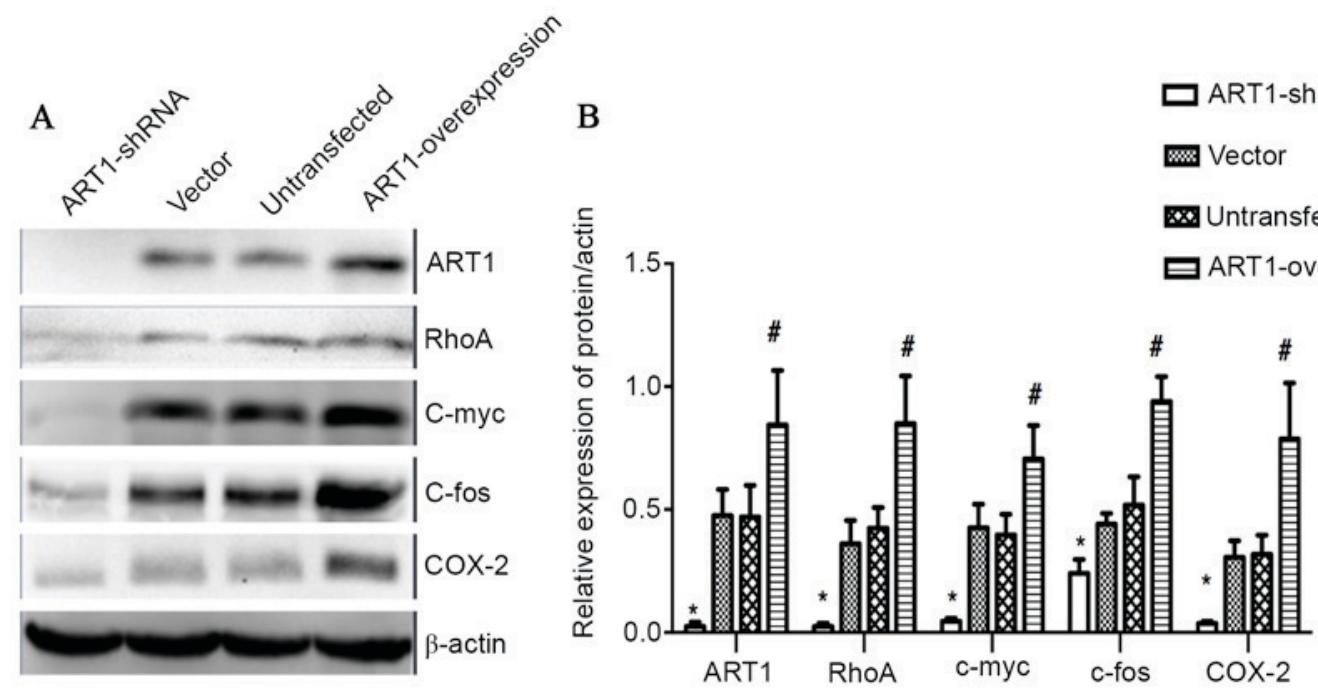

Figure 5. Effect of ART1 on the expression levels of RhoA, c-myc, c-fos and COX-2 in subcutaneously transplanted CT26 tumor tissue of BALB/c mice. (A) A representative western blot showing the expression levels of ART1, RhoA, c-myc, c-fos, COX-2 in subcutaneously transplanted CT26 tumor tissue in BALB/c mice. (B) Quantitative analysis revealed that the expression levels of ART1, RhoA, c-myc, c-fos and COX-2 in subcutaneously transplanted ART1-overexpression CT26 tumors were increased. However, the expression levels of ART1, RhoA, c-myc, c-fos and COX-2 in subcutaneously transplanted ART1-shRNA CT26 tumors were decreased. "P $<0.05$, ART1-shRNA group vs. vector and untransfected groups; ${ }^{*} \mathrm{P}<0.05$, ART1-overexpression group vs. vector and untransfected groups. ART1, ADP-ribosyltransferase 1; RhoA, Ras homolog gene family member A; COX-2, cyclooxygenase-2; ART1-shRNA, ART1-short hairpin RNA.

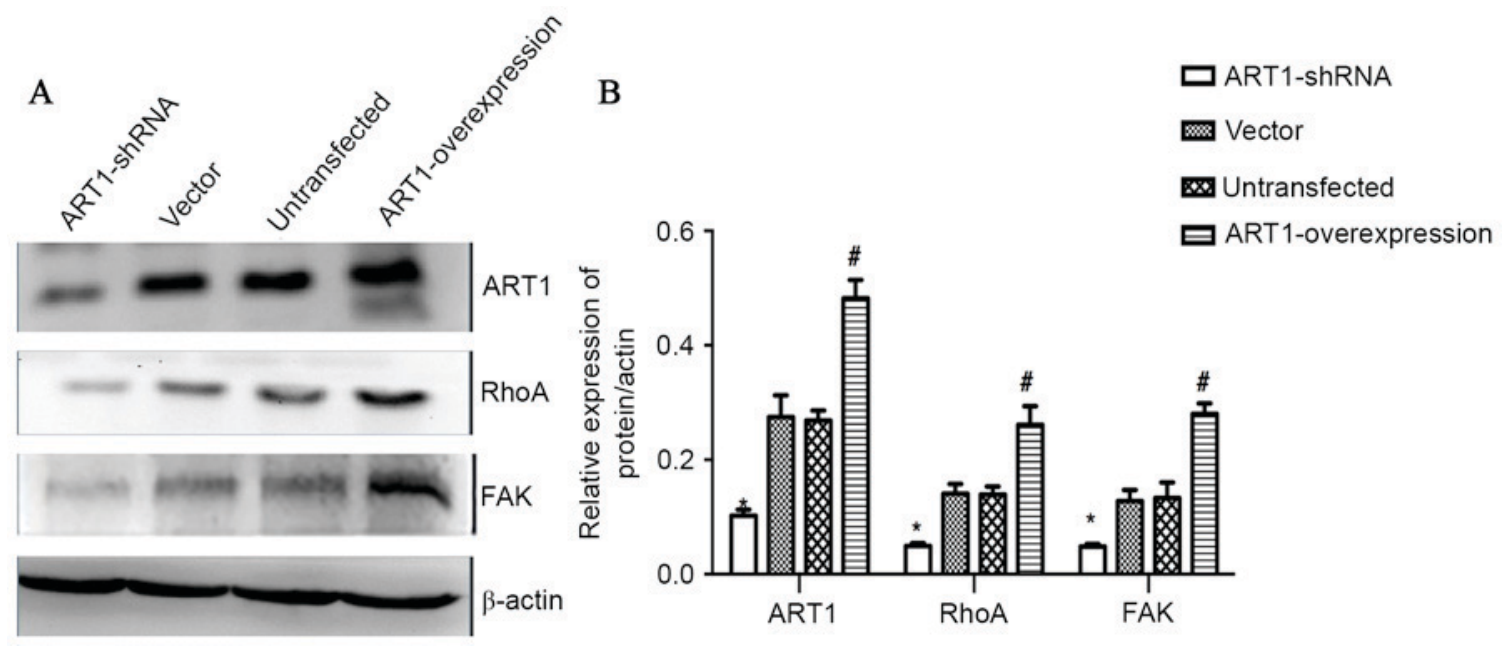

Figure 6. Effect of ART1 on the expression levels of RhoA and FAK in spleen-transplanted CT26 tumor tissue of BALB/c mice. (A) A representative western blot showing the expression levels of ART1, RhoA and FAK in spleen-transplanted CT26 tumor tissue of BALB/c mice. (B) Quantitative analysis revealed that the expression levels of ART1, RhoA and FAK in spleen-transplanted ART1-overexpression CT26 tumor were increased. However, the expression levels of ART1, RhoA, c-myc, c-fos and COX-2 in spleen-transplanted ART1-shRNA CT26 tumor were decreased. "P<0.05, ART1-shRNA group vs. vector and untransfected groups; "P<0.05, ART1-overexpression group vs. vector and untransfected groups. ART1, ADP-ribosyltransferase 1; RhoA, Ras homolog gene family member A; FAK, focal adhesion kinase; COX-2, cyclooxygenase-2; ART1-shRNA, ART1-short hairpin RNA.

tissue of BALB/c mice. The expression levels of ART1, RhoA, c-myc, c-fos and COX-2 in subcutaneously transplanted ART1-overexpression CT26 tumors were all higher than those in the control groups $(\mathrm{P}<0.05)$. However, the expression levels of ART1, RhoA, c-myc, c-fos and COX-2 in subcutaneously transplanted ART1-shRNA CT26 tumors were lower than in those in the control groups $(\mathrm{P}<0.05)$. No significant differences were identified between the vector-control and untransfected groups ( $\mathrm{P}>0.05$; Fig. 5A and B).

Effect of ART1 on the expression levels of RhoA and FAK in spleen-transplanted CT26 tumor tissue of BALB/c mice. Compared with the vector-control and untransfected groups, the expression levels of ART1, RhoA and FAK in spleen transplanted ART1-shRNA CT26 tumors were decreased, and the expression levels of these proteins in spleen-transplanted ART1-overexpression CT26 tumors were increased $(\mathrm{P}<0.05)$. No significant differences were identified between the expression levels of these proteins in the vector-control and untransfected groups ( $\mathrm{P}>0.05$; Fig. $6 \mathrm{~A}$ and $\mathrm{B})$.

\section{Discussion}

Yau et al (6) hypothesized that mono-ADP-ribosylation enzymes may be associated with the progression of gastric cancer. A previous study (11) has shown that ART1 expression 
was increased in colorectal cancer and has a positive correlation with the expression of vascular endothelial growth factor (VEGF), which suggests that it may have an association with tumor angiogenesis. It has also been observed that the silencing of ART1 in CT26 cells may inhibit the proliferation of cells by restraining cell cycle at the G0/G1 phase, suppressing matrix adhesion and migration in vitro (12-14). However, whether the changes of ART1 in CT26 cells are able to affect the proliferation and invasion in vivo has yet to be fully elucidated. The present study demonstrated the reduction in the volume and weight of subcutaneously transplanted ART1-shRNA CT26 tumor tissue in BALB/c mice. However, there was an increase in the volume and weight of subcutaneously transplanted ART1-overexpression CT26 tumor tissue in BALB/c mice. The results also demonstrated that a reduction in the volume and weight of spleen-transplanted CT26 tumor tissue occurred in BALB/c mice with the silencing of ART1 in CT26 cells, and an increase with the overexpressing of ART1 in CT26 cells. The average survival time of BALB/c mice with subcutaneously transplanted CT26 tumors or spleen transplanted CT26 tumors was significantly shortened with the overexpression of ART1, and was extended with the silencing of ART1. Taken together, these data demonstrated that ART1 may affect the growth and development of transplanted CT26 tumor in vivo.

In skeletal muscle cells, ART1 catalyzes the modification of mono-ADP-ribosylation on integrin $\alpha 7 \beta 1$, which may promote the binding of integrin and laminin and lead to the activation of FAK and of Rho, resulting in the formation of stress fibers and the shrinkage of cells (15-18). Integrin $\beta 1$, an important signaling molecule on the cell membrane, is able to associate with a variety of intracellular signaling molecules, including FAK, Rho and integrin-linked kinase (ILK) (19). It has been suggested that phosphorylation of the Rho effector may also be inhibited by an appropriate amount of MIBG (5). The same study also hypothesized that arginine-specific mono-ADP-ribosylation is involved in a Rho-dependent signaling pathway. In the present study, expression levels of FAK and RhoA decreased in the ART1-shRNA group, whereas they increased in the ART1=overexpression group. Therefore, the change in the levels of ART1 may exert an influence on the FAK and RhoA signaling pathways in colon carcinoma.

FAK is known as a regulator of cell migration. Schaller (20) demonstrated that enhanced FAK signaling may promote cell motility, whereas inhibited FAK signaling could suppress cell migration. Sieg et al (18) demonstrated that integrin $\beta 1-F A K$ is inactive in non-metastatic cancer cells, whereas it exhibits strong activity in metastatic cancer cells. Silencing integrin $\beta 1$ could control the activity of FAK and further promote cell migration. The small G-protein, RhoA, also may mediate the RhoA/Rho-kinase (ROCK) and FAK signaling pathways, and have a marked effect on tumor cell migration $(18,21)$. The present study demonstrated that expression levels of RhoA and FAK in spleen-transplanted CT26 tumors were decreased significantly due to ART1 gene silencing, and increased with ART1 overexpression in CT26 cells.

RhoA, a member of the Rho GTPase family, has been known to regulate the actin cytoskeleton in the formation of stress fibers (22), cytoskeletal dynamics, gene transcription, cell-cycle progression and cell transformation (23). ROCK is an important downstream effector of RhoA. It has been demonstrated that the RhoA/ROCK pathway serves an important role in various fundamental cellular functions, including proliferation (24). The c-myc proto-oncogene is an important regulator of cell proliferation, growth and differentiation (25). Kamaraju and Roberts (26) indicated that inhibition of Rho/ROCK activity is required for downregulation of the expression levels of c-myc protein, and the subsequent suppression of the growth of breast cancer cells. The Rho-ROCK-c-myc cascade partly contributes to vascular endothelial growth factor induction by lysophosphatidic acid in ovarian cancer (27). C-myc silencing not only efficiently downregulates the expression of c-myc, but also inhibits the proliferation of HT-29 cells and suppresses the growth of colon cancer cells in vivo (28). Rho is also involved in the shear-stress induction of c-fos (29) and may stimulate the expression levels of c-fos (30). The inhibition of ROCK activity, and the subsequent disruption of actin filaments, may induce a decrease in c-fos activity (29). C-fos-siRNA attenuated the invasive ability of Lovo cells (31) and the growth of human colon carcinoma cells in athymic mice (32). C-fos, the dysregulation of which may lead to the development of cancer, is involved in important cellular events, including cell proliferation, differentiation and survival (33). RhoA may promote the expression of COX-2 via a mechanism dependent on the transcription factor, nuclear factor- $\kappa \mathrm{B}$ (34). The inhibition of COX-2 may suppress the growth of HCA-7 and Moser-S colon cancer cells (35). Increased COX-2 activity has a positive effect on the progression of colorectal cancer (36). The present study demonstrated that the expression levels of RhoA and the downstream factors, c-myc, c-fos, and COX-2 proteins, were decreased significantly in vivo due to ART1 gene silencing, and increased with ART1 overexpression in CT26 cells. Thus, it has been demonstrated that the effect of ART1 on the proliferation of CT26 cells may be associated with RhoA and its downstream signal-transduction pathway.

Thus, ART1 serves a facilitatory role in the proliferation and migration of CT26 cells in vivo, and this effect may be associated with the factors downstream of FAK and RhoA, c-myc, c-fos, and COX-2. However, the underlying mechanisms require further investigation.

\section{Acknowledgements}

The present study was supported by the Ministry of Education Specialized Research Fund for the Doctoral Program of Higher Education (grant no. 20105503110009), the Science and Technology Program of Chongqing Municipal Education Commission (grant no. KJ110322) and the National Nature Science Foundation of China (grant no: 30870946).

\section{References}

1. Hassa PO, Haenni SS, Elser M and Hottiger MO: Nuclear ADP-ribosylation reactions in mammalian cells: Where are we today and where are we going? Microbiol Mol Biol Rev 70: 789-829, 2006.

2. Laing S, Unger M, Koch-Nolte F and Haag F: ADP-ribosylation of arginine. Amino Acids 41: 257-269, 2011.

3. Stevens LA, Levine RL, Gochuico BR and Moss J: ADP-ribosylation of human defensin HNP-1 results in the replacement of the modified arginine with the noncoded amino acid ornithine. Proc Natl Acad Sci USA 106: 19796-19800, 2009. 
4. Corda D and Di Girolamo M: Functional aspects of protein mono-ADP-ribosylation. EMBO J 22: 1953-1958, 2003.

5. Yau L, Litchie B, Thomas S, Storie B, Yurkova N and Zahradka P: Endogenous mono-ADP-ribosylation mediates smooth muscle cell proliferation and migration via protein kinase $\mathrm{N}$-dependent induction of c-fos expression. Eur J Biochem 270: 101-110, 2003.

6. Yau L, Molnar P, Moon MC, Buhay S, Werner JP, Molnar K, Saward L, Rizzo DD and Zahradka P: Meta-iodobenzylguanidine, an inhibitor of arginine-dependent mono(ADP-ribosyl)ation, prevents neointimal hyperplasia. J Pharmacol Exp Ther 326 717-724, 2008.

7. Tang Y, Wang YL, Yang L, Xu JX, Xiong W, Xiao M and Li M: Inhibition of arginine ADP-ribosyltransferase 1 reduces the expression of poly(ADP-ribose) polymerase-1 in colon carcinoma. Int J Mol Med 32: 130-136, 2013.

8. Kuang J, Wang YL, Xiao M, Tang Y, Chen WW, Song GL, Yang X and Li M: Synergistic effect of arginine-specific ADP-ribosyltransferase 1 and poly(ADP-ribose) polymerase- 1 on apoptosis induced by cisplatin in CT26 cells. Oncol Rep 31: $2335-2343,2014$.

9. Huang W, Wu YL, Zhong J, Jiang FX, Tian XL and Yu LF: Angiotensin II type 1 receptor antagonist suppress angiogenesis and growth of gastric cancer xenografts. Dig Dis Sci 53: 1206-1210, 2008.

10. Liu HY, Huang ZL, Yang GH, Lu WQ and Yu NR: Inhibitory effect of modified citrus pectin on liver metastases in a mouse colon cancer model. World J Gastroenterol 14: 7386-7391, 2008.

11. Yang L, Wang YL, Sheng YT, Xiong W, Xu JX, Tang Y and Li X: The correlation of ART1 expression with angiogenesis in colorectal carcinoma and it relationship with VEGF and integrin aVß3 expressions. Basic Clin Med 9: 1064-1069, 2012.

12. Xiao M, Tang Y, Wang YL, Yang L, Li X, Kuang J and Song GL: ART1 silencing enhances apoptosis of mouse CT26 cells via the PI3K/Akt/NF-kB pathway. Cell Physiol Biochem 32: 1587-1599, 2013.

13. Xiong W, Tang Y, Wang YL and Xu JX: Effects of ART1 gene silencing on the ability of CT26 cellular matrix adhesion and migration. Fudan Univer J Med Sci 40: 328-334, 2013.

14. Xu JX, Wang YL, Tang Y and Xiong W: Effect of ART1 gene silencing by RNA interference on the proliferation of mouse colon carcinoma cells and its possible mechanism. Tumor 32: 949-954, 2012.

15. Sawhney RS, Liu W and Brattain MG: A novel role of ERK5 in integrin-mediated cell adhesion and motility in cancer cells via Fak signaling. J Cell Physiol 219: 152-161, 2009.

16. Zhao Z, Gruszczynska-Biegala J and Zolkiewska A: ADP-ribosylation of integrin alpha7 modulates the binding of integrin alpha7beta1 to laminin. Biochem J 385: 309-317, 2005.

17. Shibue $\mathrm{T}$ and Weinberg RA: Integrin beta 1-focal adhesion kinase signaling directs the proliferation of metastatic cancer cells disseminated in the lungs. Proc Natl Acad Sci USA 106: 10290-10295, 2009

18. Sieg DJ, Hauck CR and Schlaepfer DD: Required role of focal adhesion kinase (FAK) for integrin-stimulated cell migration. J Cell Sci 112: 2677-2691, 1999.

19. Gilcrease MZ: Integrin signaling in epithelial cells. Cancer Lett 247: 1-25, 2007.

20. Schaller MD: Cellular functions of FAK kinases: Insight into molecular mechanisms and novel functions. J Cell Sci 123: $1007-1013,2010$
21. Dorfleutner A, Stehlik C, Zhang J, Gallick GE and Flynn DC AFAP-110 is required for actin stress fiber formation and cell adhesion in MDA-MB-231 breast cancer cells. J Cell Physiol 213: 740-749, 2007.

22. Nobes CD and Hall A: Rho, rac, and cdc42 GTPases regulate the assembly of multimolecular focal complexes associated with actin stress fibers, lamellipodia, and filopodia. Cell 81: 53-62, 1995.

23. Zhang S, Tang Q, Xu F, Xue Y, Zhen Z, Deng Y, Liu M, Chen J, Liu S, Qiu M, et al: RhoA regulates G1-S progression of gastric cancer cells by modulation of multiple INK4 family tumor suppressors. Mol Cancer Res 7: 570-580, 2009.

24. Zohrabian VM, Forzani B, Chau Z, Murali R and Jhanwar-Uniyal M: Rho/ROCK and MAPK signaling pathways are involved in glioblastoma cell migration and proliferation. Anticancer Res 29: 119-123, 2009.

25. Levens DL: Reconstructing Myc. Gene Dev 17: 1071-1077, 2003.

26. Kamaraju AK and Roberts AB: Role of Rho/ROCK and p38 MAP kinase pathways in transforming growth factor-beta-mediated Smad-dependent growth inhibition of human breast carcinoma cells in vivo. J Biol Chem 280: 1024-1036, 2005.

27. Song Y, Wu J, Oyesanya RA, Lee Z, Mukherjee A and Fang X: Sp-1 and c-Myc mediate lysophosphatidic acid-induced expression of vascular endothelial growth factor in ovarian cancer cells via a hypoxia-inducible factor-1-independent mechanism. Clin Cancer Res 15: 492-501, 2009.

28. Zhang X, Ge YL and Tian RH: The knockdown of c-myc expression by RNAi inhibits cell proliferation in human colon cancer HT-29 cells in vitro and in vivo. Cell Mol Biol Lett 14: 305-318, 2009.

29. Shiu YT, Li S, Yuan S, Wang Y, Nguyen P and Chien S: Shear stress-induced c-fos activation is mediated by Rho in a calcium-dependent manner. Biochem Biophys Res Commun 303: $548-555,2003$

30. Ueyama T, Sakoda T, Kawashima S, Hiraoka E, Hirata KI, Akita $\mathrm{H}$ and Yokoyama M: Activated RhoA stimulates c-fos gene expression in myocardial cells. Circ Res 81: 672-678, 1997.

31. Jia ZC, Wan YL, Tang JQ, Dai Y, Liu YC, Wang X and Zhu J: Tissue factor/activated factor VIIa induces matrix metalloproteinase-7 expression through activation of c-Fos via ERK1/2 and p38 MAPK signaling pathways in human colon cancer cell. Int J Colorectal Dis 27: 437-445, 2012.

32. Pandey MK, Liu G, Cooper TK and Mulder KM: Knockdown of c-Fos suppresses the growth of human colon carcinoma cells in athymic mice. Int J Cancer 130: 213-222, 2012.

33. Tulchinsky E: Fos family members: Regulation, structure and role in oncogenic transformation. Histol Histopathol 15: 921-928, 2000.

34. Benitah SA, Valerón PF and Lacal JC: ROCK and nuclear factor-kappaB-dependent activation of cyclooxygenase-2 by Rho GTPases: Effects on tumor growth and therapeutic consequences. Mol Biol Cell 14: 3041-3054, 2003.

35. Fosslien E: Biochemistry of cyclooxygenase (COX)-2 inhibitors and molecular pathology of COX-2 in neoplasia. Crit Rev Cl Lab Sci 37: 431-502, 2000

36. Asting AG, Carén H, Andersson M, Lönnroth C, Lagerstedt K and Lundholm K: COX-2 gene expression in colon cancer tissue related to regulating factors and promoter methylation status. BMC Cancer 11: 238, 2011. 